\title{
Experimental investigation and modelling of the curing behaviour of photopolymers
}

\author{
T. Rehbein ${ }^{a, *}$, A. Lion ${ }^{a}, \quad$ M. Johlitz ${ }^{a}$ and A. Constantinescu ${ }^{b}$ \\ ${ }^{a}$ Institute of Mechanics, Faculty of Aerospace Engineering, Bundeswehr University Munich, Werner-Heisenberg-Weg 39 , 85577 \\ Neubiberg, Germany \\ ${ }^{b}$ Laboratoire de Mecanique des Solides, CNRS, École Polytechnique, Institut Polytechnique de Paris, Route de Saclay, 91128 \\ Palaiseau, France
}

\section{ARTICLE INFO}

Keywords:

Photopolymer

Photo-DSC

Digital Light Processing

Stereolithography

Reaction kinetics

Additive manufacturing

\begin{abstract}
A B S T R A C T
This paper focuses on the experimental investigation and modelling of the crosslinking reaction of photopolymers used in additive manufacturing processes such as digital light processing and stereolithography. Starting with general mathematical concepts for the description of the material behaviour of polymeric materials, the importance of modelling the crosslinking reaction is emphasized. In order to characterise the crosslinking reaction experimentally, photocalorimetric measurements with varying isothermal temperature and light intensity are shown. From the exothermic heat flows measured during the crosslinking reaction, the degree of cure can be determined for each experimental scenario as a function of time. It is shown that the test temperature and light intensity have a significant influence on the crosslinking reaction. A modelling approach for the description of the crosslinking reaction incorporating temperature and light intensity is presented. Moreover, parameter identification and a comparison of the proposed model with the experiments are conducted. The indentified model has an excellent match with experimental data, resulting in a least square error smaller than $3 \%$. The proposed model and identification method opens up several extensions for the modelling of the material behaviour.
\end{abstract}

\section{Introduction}

Additive manufacturing (AM) is an innovative technology to create three dimensional objects with complex geometry. A large panel of different technologies and materials ensures a maximum design freedom for the production of polymeric and metallic components leading to a reduced time and lowered expenses for the development of new products to be saled.

Current studies in the additive manufacturing of polymers cover, for example, the usage of additively manufactured lattice structures as energy-absorbing helmet liners for improved blunt impact protection [1]. Generally, additive manufacturing enables the fabrication of novel designs consisting of arbitrary arranged lattice structures for energy absorbing components with respect to lightweight construction requirements [2]. Using these technologies, components can be manufactured not only at the macro scale but also down to the nano scale including the addition of fillers [3].

Furthermore, dental applications such as customised crowns, implants and dentures for patients can be made precisely with additive manufacturing technologies [4]. Although the technology has been used in industrial applications since the 1980s, reliable and experimentally validated models for the design of printed components are still missing. Especially the material modelling and finite element analysis of photopolymers used

\footnotetext{
* Corresponding author

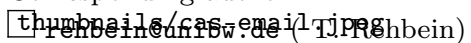

ORCID(s): 0000-0003-0833-2264 ( T. Rehbein)
}

in additive manufacturing processes such as digital light processing and stereolithography are challenging tasks. For this purpose, the crosslinking reaction in the additive manufacturing process must be described correctly by a phenomenological model and adapted to experimental data.

Many developers of commercial software solutions for the numerical analysis of structures (e.g. Ansys Inc., Altair Engineering Inc., Dassault Systèmes SE) are also working on numerical tools for the analysis of additive manufacturing processes, see $[5]$ and [6]. These include process simulation, part analysis and topology optimisation, just to name a few fields of application. All of the aforementioned software developers have one thing in common: they need experimentally based models for the materials which are analysed in their programs for additive manufacturing. Instead of the countless printing of a desired geometry with variation of the process parameters up to the desired product properties, the printing process can be optimised on the computer with experimentally validated simulation methods even before the final printing. This paper starts with this goal.

The crosslinking of photopolymers is initiated by the absorption of light in the UV wavelength range. Commercially available 3D printers using photopolymer resins work typically at wavelenghts of $365 \mathrm{~nm}$ (atum3D DLP-Station 5-365) and $405 \mathrm{~nm}$ (Formlabs Form 3), respectively. Besides that, the process depends strongly on the temperature: a higher ambient 


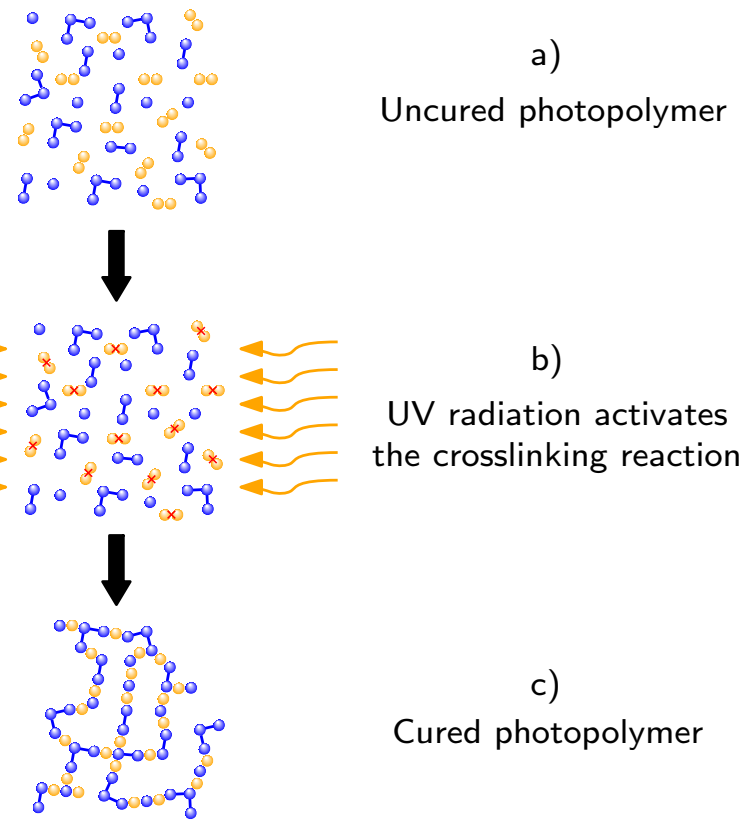

Figure 1: Simplified sketch of the crosslinking progress of a photopolymer based on [7].

temperature leads to faster crosslinking whereby the same applies to an increasing light intensity.

Fig. 1 shows a simplified sketch of the crosslinking progress of a photopolymer. Photopolymers usually consist of monomers (0), oligomers ( $\infty, \infty$ ) and photoinitiators ( $\infty$ ) whereby it is also possible to design photopolymer resins using renewable raw materials [8]. At first, the components of the liquid photopolymer are not crosslinked (a). The photoinitiators are excited by the incoming UV radiation (b) and finally crosslink with the monomers and oligomers (c) to a solid material.

Digital light processing and stereolithography are two similar additive manufacturing technologies using photopolymerisation. The former uses a projector as a light source and a complete layer is cured simultaneously whereby the latter one uses a laser as a light source which irradiates the trajectory to be printed in each individual layer.

The phenomenological modelling of the transition from a liquid to a solid polymeric material has often been used to describe the hot curing of adhesives. Many authors developed material models for the description of the curing behaviour of adhesives and adapted their models to experimental data, see [9] and [10]. These models are primarily based on the work of [11] and [12]. Furthermore, in [13], a material model for the description of the crosslinking reaction of acrylic bone cements is developed and adapted to experimental data.

In [14], Mahnken developed a macroscopic material model incorporating temperature dependent viscoelastic effects during curing processes. As a numerical example, the prognosis ability of the model is shown us- ing a deep drawing process. Based on these findings, Mahnken and Dammann presented a multiscale approach for the curing of epoxy resin systems in the resin transfer moulding process, see [15].

To get a broad knowledge, the interested reader is referred to [16] in which a highly detailed overview is given about the modelling of curing processes of polymers.

Tryson and Shultz [17] were the first to develop a test method for the investigation of the crosslinking reaction of photopolymers used in coating applications with the help of a modified DSC device. In addition, empirical model equations for curing were derived and adapted to the experimental data.

Maffezzoli and Terzi [18] investigated the influence of light intensity on the crosslinking reaction of a photopolymer used in stereolithography, but without investigating temperature dependence. Like Tryson and Shultz, they developed a test method using DSC with a xenon lamp to study the crosslinking reaction.

In [19], a simulation methodology based on the finite element method was developed for the propagation of light in liquid photopolymer resins used in stereolithography. The program also includes a material model for the description of the degree of cure taking into account light intensity, temperature and photoinitiator concentration. This study is a purely theoretical/numerical one and does not include an experimental investigation of the crosslinking reaction of photopolymers used in additive manufacturing.

$\mathrm{Wu}$ et al. investigate the evolution of mechanical properties during photopolymerisation and build up a model for the finite element analysis of residual stresses and bending strains, see [20].

The experimental investigation of different postcuring processes and their influence on the thermal and mechanical properties of a commercially available photopolymer is the main part of [21]. Calorimetric measurements and thermogravimetric as well as dynamic mechanical analyses are conducted incorporating two different postcuring mechanisms (with and without UV light exposure) and several curing times for printed specimens.

Calorimetric measurements with the aid of an additional light source proved useful for the experimental investigation of the crosslinking reaction of photopolymers [22]. Since the degree of cure directly influences the mechanical, thermal and chemical properties of photopolymers, a validated material model for the degree of cure is essential for structural computations of additive manufacturing processes. 


\section{Material modelling and reaction kinetics}

\subsection{Representation by rheological elements}

In phenomenological modelling of viscoelastic materials, it is common practice using an arrangement of standardised rheological elements to represent the material behaviour of polymers [23]. Fig. 2 shows the rheological model for a viscoelastic polymeric material consisting of mechanical (mech), thermal (th) and chemical (ch) parts. The mechanical part consists of an arrangement of springs ( $-M-$ ) and dashpots (고) which is also called the generalised Maxwell model representing the time dependent material behaviour. Both shear moduli $G_{k}$ and viscosities $\eta_{k}$ can depend on temperature $\theta$ and degree of cure $q$ which works as an internal variable. The number of Maxwell elements $N$ is arbitrary and depends on the desired representation quality of the viscoelastic spectrum. The rate-independent behaviour is represented by the equilibrium shear modulus $G_{\infty}$ and bulk modulus $K$. In addition, the bulk behaviour is supposed to be purely elastic. For small deformations, the total three dimensional strain $\boldsymbol{E}$ is split additively into mechanical, thermal and chemical parts [24]:

$$
\boldsymbol{E}=\boldsymbol{E}_{\mathrm{mech}}+\boldsymbol{E}_{\mathrm{th}}+\boldsymbol{E}_{\mathrm{ch}}
$$

For the consideration of curing processes at large deformations, Lion and Höfer [25] and Sain et al. [26] proposed a general finite strain framework for the formation of the polymer network in a stress-free intermediate configuration.

The mechanical part $\boldsymbol{E}_{\text {mech }}$ is again divided into a purely elastic $\boldsymbol{E}_{\text {elk }}$ and a viscous part $\boldsymbol{E}_{\text {ink }}$ for each Maxwell element $k$ :

$$
\boldsymbol{E}_{\text {mech }}=\boldsymbol{E}_{\mathrm{elk}}+\boldsymbol{E}_{\text {ink }} \quad \text { with } \quad k=1, \ldots, N
$$

For the inelastic strains $\boldsymbol{E}_{\text {ink }}, N$ evolution equations are proposed [27]:

$\dot{\boldsymbol{E}}_{\mathrm{ink}}=\frac{1}{\eta_{k}(\theta, q)}\left(2 G_{k}(\theta, q) \boldsymbol{E}_{\mathrm{elk}}^{\mathrm{D}}\right) \quad$ with $\quad k=1, \ldots, N$

Here, $(.)^{\mathrm{D}}=()-.\frac{1}{3} \operatorname{tr}(.) \mathbf{1}$ denotes the deviatoric part of a second order tensor and $\mathbf{1}$ is the unit second order tensor.

If the material is exposed to an increase in temperature, it expands. This mechanism is represented by the symbol $\square$ and described mathematically by isotropic thermal expansion:

$$
\boldsymbol{E}_{\text {th }}=\alpha_{\text {th }}(q) \Delta \theta \mathbf{1}
$$

Generally, the coefficient of thermal expansion (CTE) $\alpha_{\text {th }}$ depends on the degree of cure $q$. The density of the

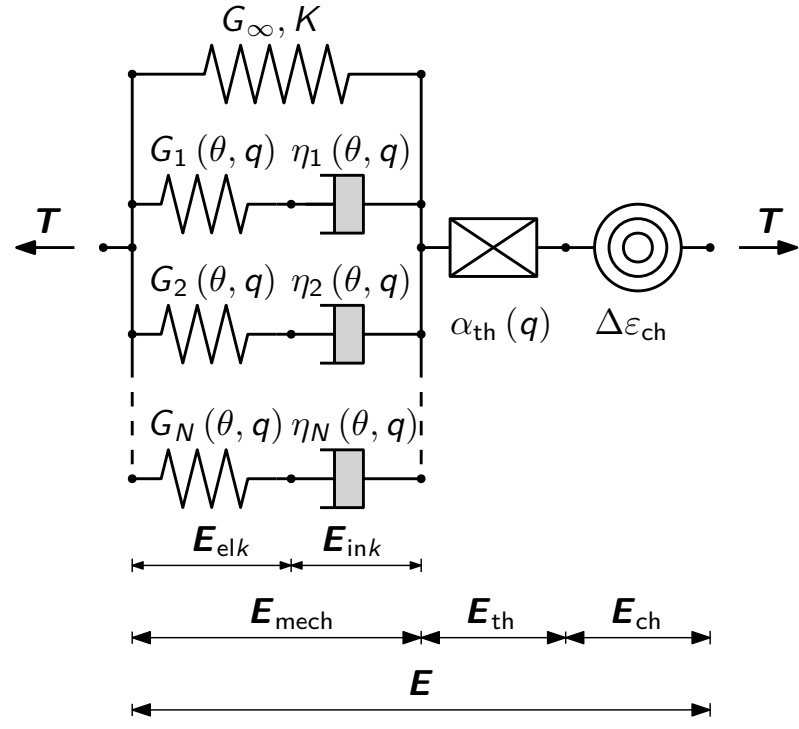

Figure 2: Rheological model for the representation of the mechanical, thermal and chemical parts of polymeric materials.

material increases during the transition from a liquid resin to a solid material due to the crosslinking reaction. Due to the constant mass, the volume is reduced. This process is also known as chemical shrinkage (-(a)-) and is assumed to be isotropic, similar to thermal expansion:

$$
\boldsymbol{E}_{\mathrm{ch}}=q \Delta \varepsilon_{\mathrm{ch}} \mathbf{1}
$$

$\Delta \varepsilon_{\text {ch }}$ is a material parameter and can be determined, for example, by measurements according to the Archimedes' principle. Chemical shrinkage can induce residual stresses which can negatively influence the desired mechanical properties of the printed product. Besides that, excessive warpage can occur during the printing process.

Thus, under the assumption of isotropy and for a homogeneous material, the resulting stress $\boldsymbol{T}$ is split into a volumetric part $\boldsymbol{T}^{\vee}$ and deviatoric part $\boldsymbol{T}^{\mathrm{D}}$ and reads as follows:

$$
\begin{aligned}
\boldsymbol{T} & =\boldsymbol{T}^{\vee}+\boldsymbol{T}^{\mathrm{D}} \\
\boldsymbol{T}^{\vee} & =K\left(\left(\boldsymbol{E}-\boldsymbol{E}_{\mathrm{th}}-\boldsymbol{E}_{\mathrm{ch}}\right): \mathbf{1}\right) \mathbf{1} \\
\boldsymbol{T}^{\mathrm{D}} & =2 G_{\infty} \boldsymbol{E}_{\text {mech }}^{\mathrm{D}}+\sum_{k=1}^{N} 2 G_{k}(\theta, q)\left(\boldsymbol{E}_{\text {mech }}^{\mathrm{D}}-\boldsymbol{E}_{\text {ink }}\right)
\end{aligned}
$$

Since the degree of cure influences the mechanical, thermal and chemical properties of the material and has a high impact on the quality of the printed product, it is advisable to start with the experimental characterisation and modelling of the crosslinking reaction of the photopolymer.

\subsection{Modelling of the degree of cure}

This contribution takes up the basic work of Kamal [12] using the consideration of light intensity as 
proposed by Maffezzoli and Terzi. In addition, the influence of the temperature is also taken into account. The ordinary differential equation for the degree of cure $q$ depends on time $t$, light intensity $\mathcal{I}(z(t), t)$ and absolute temperature $T$. Following the work of Kamal, an autocatalytic model of $(m+n)$ th order incorporating the maximum attainable degree of cure $q_{\max }$ is applied for the degree of cure $q$ :

$$
\begin{aligned}
\dot{q}= & \left(k_{1}(\mathcal{I}(z(t), t), T)+k_{2}(\mathcal{I}(z(t), t), T) q^{m}\right) \\
& \cdot\left(q_{\max }-q\right)^{n}
\end{aligned}
$$

Here, $\mathcal{I}(z(t), t)$ denotes the time- and location-dependent light intensity. The light intensity must depend on the vertical location $z(t)$ since it evolves during the printing process. The maximum attainable degree of cure $q_{\text {max }}$ acts as a limit value. The functions

$$
k_{i}(\mathcal{I}(z(t), t), T)=k_{0 i}(T)\left(\frac{\mathcal{I}(z(t), t)}{\mathcal{I}_{\text {ref }}}\right)^{b}
$$

with the Arrhenius type equations

$$
k_{0 i}(T)=A_{i} \exp \left(-\frac{E_{i}}{R T}\right)
$$

are based on the proposal by Maffezzoli and Terzi [18] depending on light intensity $\mathcal{I}(z(t), t)$ and temperature $T$. If no light source is present, i.e. $\mathcal{I}(z(t), t)=0$, the crosslinking reaction is stopped. $A_{i}$ and $E_{i}$ are pre-exponential factors and activation energies, respectively. $R$ denotes the universal gas constant with $R=$ $8.314 \mathrm{~J} /(\mathrm{mol} \mathrm{K})$. The additional parameter $b$ is introduced for a better representation of experimental data. Furthermore, the auxiliary constant $\mathcal{I}_{\text {ref }}=1 \mathrm{~mW} / \mathrm{cm}^{2}$ is used to convert the light intensity into an unitless value.

Both isothermal and non-isothermal processes as well as time dependent light intensity boundary conditions can be taken into account by this model.

\section{Experimental investigation of the curing behaviour}

\subsection{Experimental setup}

In order to understand and investigate the crosslinking process of commercial photopolymers used in additive manufacturing processes, differential photocalorimetry (DPC) measurements are conducted using the TA Instruments DSC Q2000 with an additional light source (OmniCure ${ }^{\circledR}$ S2000). The light source contains a high pressure $200 \mathrm{~W}$ mercury lamp with a spectral output of 320 to $500 \mathrm{~nm}$. UV radiation is conducted directly into the DSC measurement cell via dual quartz light guides. The calibration of the light intensity at the reference and sample platforms is carried out via the separate height adjustment of the light guides and neutral density filters inside the light guides. The Tzero ${ }^{\mathrm{TM}}$

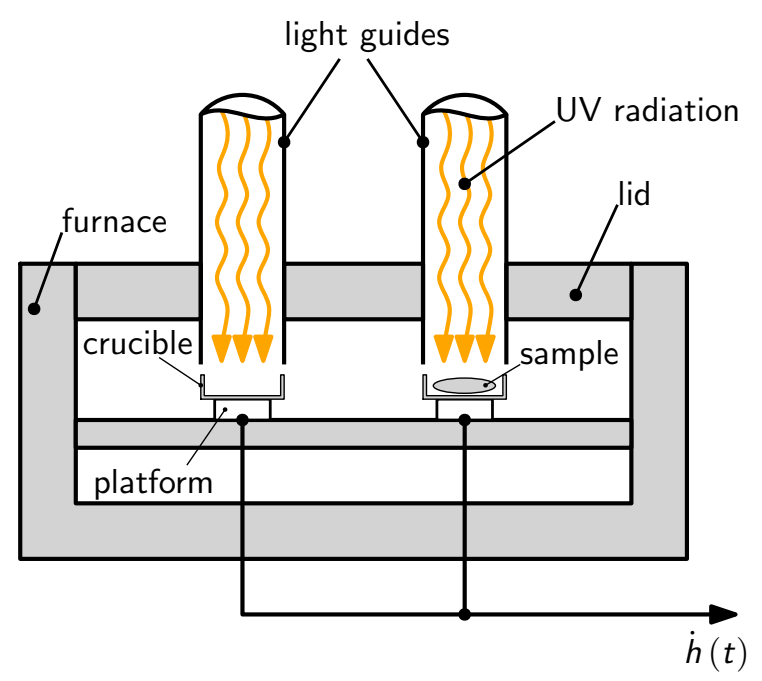

Figure 3: Schematic diagram of a photo-DSC measurement cell.

technology allows the direct measuring of the light intensity at the reference and sample platforms.

For a better understanding, fig. 3 shows a schematic diagram of a photo-DSC: the light guides point to the reference and sample crucibles made of aluminium and thus enable the crosslinking reaction of the liquid photopolymer in the sample crucible. During irradiation, the photo-DSC measures the incoming or outcoming specific heat flow $\dot{h}(t)$ of a sample located in a crucible with regard to an empty reference crucible. The quantity $\dot{h}(t)$ is related to the mass of the sample and an indicator of the degree of cure due to the exothermic crosslinking reaction.

The following relationship is used to convert the specific heat flow $\dot{h}(t)$ into the degree of cure $q(t)$ :

$$
q(t)=\frac{\int_{0}^{t} \dot{h}(\tilde{t}) \mathrm{d} \tilde{t}}{h_{\mathrm{tot}}}
$$

To put it simply, the degree of cure is the accumulated area between the adjusted photo-DSC signal and the abscissa relative to the total specific heat of reaction. Due to the normalisation to the total specific heat of reaction $h_{\text {tot }}$ during the crosslinking reaction, the degree of cure assumes values between 0 and 1 .

The commercial photopolymer LOCTITE ${ }^{\circledR}$ 3D 3830 resin was used for the photocalorimetric measurements. It is an acrylic compound for prototype development with low elongation at break, high tensile strength and low chemical shrinkage. Both stereolithography and digital light processing 3D printers can work with this photopolymer.

During the photocalorimetry measurement, following steps are performed subsequently for the determination of the specific heat flow $\dot{h}(\tilde{t})$ in eq. (12):

1. Equilibration of the measurement cell at a specific isothermal temperature $\left(-10^{\circ} \mathrm{C} \ldots 50^{\circ} \mathrm{C}\right)$. 
Table 1

Maximum attainable degree of cure $q_{\max }$ of all measurements

\begin{tabular}{r|c|c} 
& $5 \mathrm{~mW} / \mathrm{cm}^{2}$ & $10 \mathrm{~mW} / \mathrm{cm}^{2}$ \\
\hline$-10{ }^{\circ} \mathrm{C}$ & 0.469 & 0.593 \\
$0{ }^{\circ} \mathrm{C}$ & 0.619 & 0.685 \\
$10{ }^{\circ} \mathrm{C}$ & 0.675 & 0.775 \\
$20{ }^{\circ} \mathrm{C}$ & 0.775 & 0.845 \\
$30{ }^{\circ} \mathrm{C}$ & 0.851 & 0.907 \\
$40{ }^{\circ} \mathrm{C}$ & 0.904 & 0.949 \\
$50{ }^{\circ} \mathrm{C}$ & 0.947 & 0.997
\end{tabular}

2. Isothermal phase of $60 \mathrm{~s}$.

3. Irradiation of the specimen for $300 \mathrm{~s}$ at constant light intensity $\left(5 \mathrm{~mW} / \mathrm{cm}^{2}\right.$ and $\left.10 \mathrm{~mW} / \mathrm{cm}^{2}\right)$.

4. Isothermal phase of $60 \mathrm{~s}$.

The experimental conditions (temperature, light intensity) are chosen in such a way that a sufficiently large database for the subsequent adaptation of the model is guaranteed. Furthermore, the second isothermal phase in step four is applied in order to achieve a clear signal for the evaluation of the experimental data.

After finishing step four, the whole procedure is repeated with the cured specimen generating the baseline. The baseline is subtracted from the heat flow signal of the first measurement to consider only the heat flow of the crosslinking reaction. Otherwise, the light energy input would be taken into account. Additionally, nitrogen is used as a purge gas with a flow rate of $50 \mathrm{ml} / \mathrm{min}$. The sample masses are between 3 and 6 mg.

\subsection{Photocalorimetric measurements}

The specific heat flows after substraction of the baseline of all measurements are shown in fig. 4. It can be clearly seen that the crosslinking reaction proceeds very quickly and that the specific heat flows have reached their maximum value after only a few seconds. Additionally, it is obvious that a higher light intensity leads to a higher maximum value in the heat flows and that a higher temperature accelerates the reaction.

Moreover, complete curing is not achieved at low temperatures and light intensities and the crosslinking reaction is stopped prematurely, cf. fig. 7. The maximum attainable degrees of cure $q_{\max }$ for all measurements are listed in table 1 . It can be seen that an almost fully cured material is achieved at a temperature of 50 ${ }^{\circ} \mathrm{C}$, regardless of the light intensity. This result must definitely be taken into account when formulating the material model for the degree of cure.

In order to determine the total specific heat of reaction $h_{\text {tot }}$ during the crosslinking reaction, an additional measurement at non-isothermal temperature is performed. During $600 \mathrm{~s}$ irradiation with $5 \mathrm{~mW} / \mathrm{cm}^{2}$, the temperature is increased from $20^{\circ} \mathrm{C}$ to $70^{\circ} \mathrm{C}$ at 5
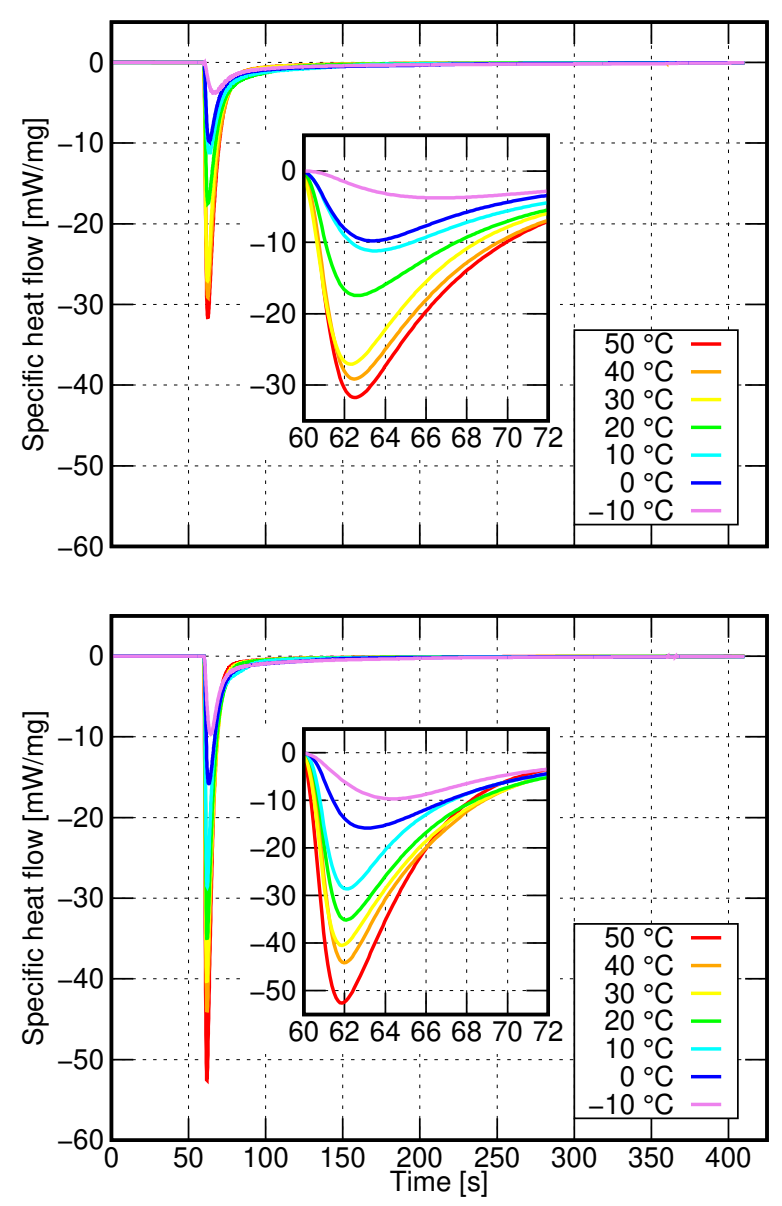

Figure 4: Measured specific heat flows after substraction of the baseline under isothermal conditions. Top: light intensity $\mathcal{I}_{1}(z(t), t)=5 \mathrm{~mW} / \mathrm{cm}^{2}$, bottom: light intensity $\mathcal{I}_{2}(z(t), t)=10 \mathrm{~mW} / \mathrm{cm}^{2}$.

${ }^{\circ} \mathrm{C} / \mathrm{min}$ (red curve in fig. 5). Subsequently, the same procedure is repeated with the cured specimen (blue curve in fig. 5).

By using this approach, the area between the red and blue curves represents the total specific heat of reaction produced during full cure. The evaluation of the area leads to a total specific heat of reaction of $h_{\text {tot }}=$ $-319.73 \mathrm{~J} / \mathrm{g}$ which is taken as reference value for the conversion of the specific heat during crosslinking reaction into degree of cure for all measurements.

Another interesting feature of the crosslinking reaction is the temperature evolution of the sample material during and after curing, see fig. 6. Initially, when the shutter of the light source is opened at $20{ }^{\circ} \mathrm{C}$ and the crosslinking reaction starts, the temperature increases briefly and then follows linear heating up to $70^{\circ} \mathrm{C}$ (red curve in fig. 6). This can also be seen in the slight inclination to the right of the specific heat flow signal of the red curve in fig. 5. In the subsequent second run (blue dotted curve in fig. 6), no temperature raise due to the opening of the shutter is recognised. 


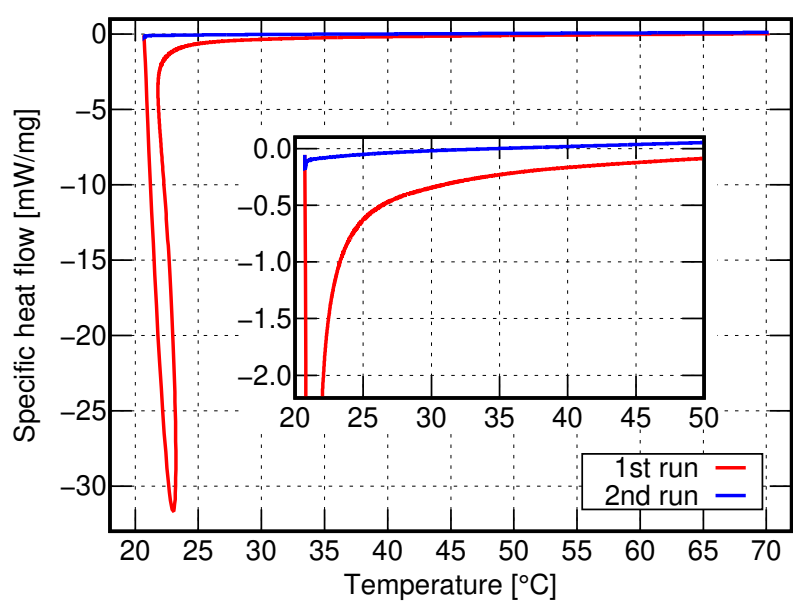

Figure 5: Photo-DSC measurement under non-isothermal conditions and additional irradiation.

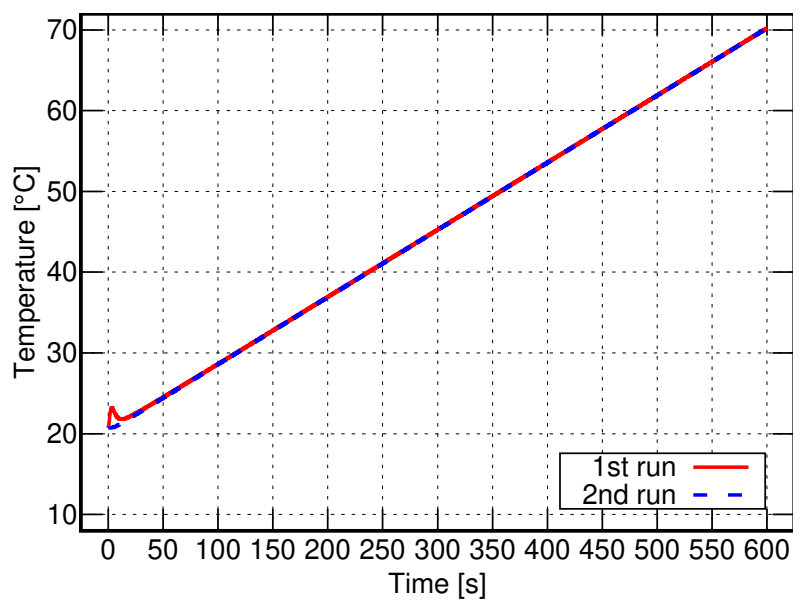

Figure 6: Measured temperature profiles of the photo-DSC measurements in fig. 5 .

\section{Results and discussion}

\subsection{Parameter identification}

The parameters in eq. (9) are identified using the commercial optimisation program LS-OPT ${ }^{\circledR}$ (Livermore Software Technology Corporation, Livermore, CA) in combination with MATLAB ${ }^{\circledR}$ 's ode45 solver for the solution of the ordinary differential equation (9). LS$\mathrm{OPT}^{\circledR}$ uses the sequential response surface methodology (SRSM) for optimisation. For further informations see [28].

In order to quantify the quality of the identified model parameters, the sum of the mean squared errors of all objective functions is calculated:

$$
\left.\mathrm{MSE}=\sum_{i=1}^{14}\left(\frac{1}{M} \sum_{j=1}^{M}\left(q_{i j, \operatorname{sim}}(\boldsymbol{x})-q_{i j, \exp }\right)\right)^{2}\right)
$$

The vector $\boldsymbol{x}$ and scalar value $M$ denote the design space vector, i.e. the parameters of the model for the
Table 2

Identified model parameters for the description of the crosslinking reaction

\begin{tabular}{c|c} 
Parameter & Identified value \\
\hline$A_{1}$ & $0.6606 \mathrm{~s}^{-1}$ \\
$A_{2}$ & $0.8744 \mathrm{~s}^{-1}$ \\
$E_{1}$ & $9794 \mathrm{~J} / \mathrm{mol}$ \\
$E_{2}$ & $1001 \mathrm{~J} / \mathrm{mol}$ \\
$b$ & 4.554 \\
$m$ & 2.996 \\
$n$ & 1.365
\end{tabular}

degree of cure $\left(A_{1}, A_{2}, E_{1}, E_{2}, b, m\right.$ and $\left.n\right)$, and the number of regression points, respectively. For the best representation of the experimental data, all 14 measurements of fig. 4 are taken into account as objective functions during the parameter identification process which is represented by the iteration index $i$. A total of $M=1000$ regression points are used for the evaluation of each mean squared error. For simplification, the particular maximum attainable degree of cure was specified as a constraint in order to achieve a better agreement with the experimental data.

\subsection{Comparison with experiments}

The parameters listed in table 2 represent the best result of the parameter identification with a summarised mean squared error of all objective functions of 0.0295 .

Fig. 7 shows a comparison between the simulations of the degree of cure according to eq. (9) with identified model parameters and all measurements conducted by the photo-DSC. For the simulations, two constant light intensities $\mathcal{I}_{1}(z(t), t)=5 \mathrm{~mW} / \mathrm{cm}^{2}$ and $\mathcal{I}_{1}(z(t), t)=10 \mathrm{~mW} / \mathrm{cm}^{2}$ as well as seven different temperatures are used as boundary conditions according to the experimental procedure which is described in sec. 3.1.

The degree of cure is evaluated by means of eq. (12) for all measurements represented in fig. 4. The proposed model is capable of predicting the temperature and light intensity dependence of the crosslinking reaction leading to a perfect representation of the experimental data. Moreover, only seven model parameters are sufficient to consider the influence of the two most important process parameters (temperature and light intensity) on the crosslinking reaction.

Both the tremendous increase to the degree of cure in the first seconds after opening of the shutter and the decrease of the conversion rate after reaching the peak are reproduced by the model in almost error-free agreement.

In dependence of the isothermal temperature, the degree of cure reaches an asymptotic value for both light intensities.

However, it is more important which light energy (light intensity multiplied by time) is applied to the 

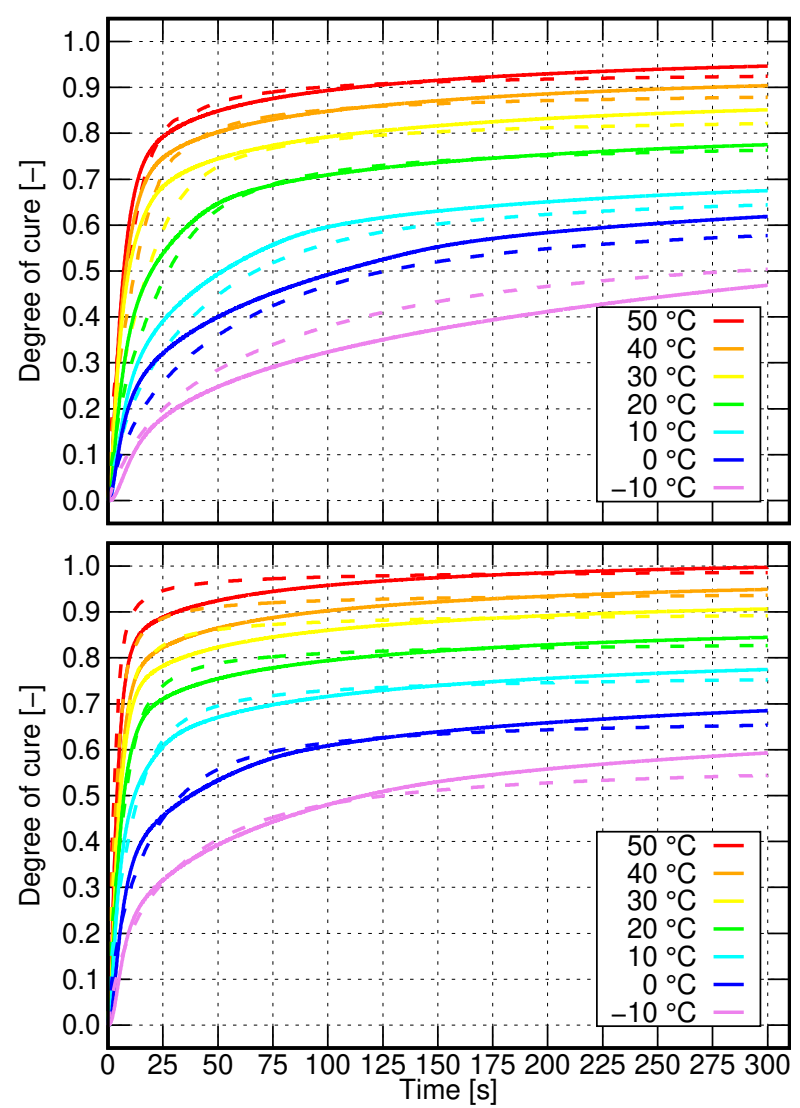

Figure 7: Measurement (-) and simulation (- -) of the degree of cure $q$ with identified model parameters. Top: light intensity $\mathcal{I}_{1}(z(t), t)=5 \mathrm{~mW} / \mathrm{cm}^{2}$, bottom: light intensity $\mathcal{I}_{2}(z(t), t)=10 \mathrm{~mW} / \mathrm{cm}^{2}$.

photopolymer during irradiation. The measurements with the lower light intensity lead to the equivalent degree of cure of the measurements with the higher light intensity when the exposure time is increased.

\section{Conclusion}

This paper discusses the experimental characterisation as well as the modelling and simulation of the crosslinking reaction of a commercial photopolymer for stereolithography and digital light processing 3D printers. Using a commercial photopolymer as sample material, it is shown that both ambient temperature and light intensity have a strong influence on the crosslinking reaction. Two conclusions can be drawn from the study of the crosslinking reaction of the photopolymer:

1. The crosslinking reaction depends strongly on the ambient temperature; the higher the temperature, the faster the crosslinking reaction and a higher degree of cure is achieved at the end of the irradiation.

2. Equivalent to the ambient temperature, an increasing light intensity also has a positive influence on the speed of the crosslinking reaction, but with a smaller effect.

The proposed model is capable of predicting the temperature and light intensity dependence of the crosslinking reaction with only seven model parameters. The combination of LS-OPT ${ }^{\circledR}$ and MATLAB ${ }^{\circledR}$ provides an excellent result for the parameter identification and a small summarised mean squared error. However, the correlation of the identified parameters with each other still has to be investigated in order to reduce the number of parameters of the model.

\section{Outlook}

In this study, the maximum attainable degree of cure $q_{\max }$ is specified as a constraint for all 14 measurements. Forthcoming considerations include the correct description of the maximum attainable degree of cure as a function of absolute temperature and light intensity:

$$
q_{\max }=f(T, \mathcal{I})
$$

The limitation of the maximum attainable degree of cure can also be described by an additional diffusion factor. For this, Fournier et al. [29] made a significant contribution which has been applied in various recent studies for epoxy resins, see [30], [31] and [32].

Since the glass transition temperature of acrylic photopolymers strongly depends on the degree of cure, conventional DSC measurements must be performed after irradiation to determine the glass transition temperature for distinct degrees of cure. Subsequently, DiBenedetto's proposal [33] can also be used to establish a relationship between the maximum attainable degree of cure and the glass transition temperature.

The dependence of the shear moduli $G_{k}(q, \theta)$ and viscosities $\eta_{k}(q, \theta)$ on the degree of cure $q$ can be determined with a dynamic shear rheometer using the same light source as with the Photo-DSC. During irradiation, a thin photopolymer layer is sheared between two plates and thereby cured. This allows the storage and loss modulus of the photopolymer to be determined experimentally, depending on the degree of cure. Afterwards, a suitable model is adapted to the experimental data to cover the mechanical properties. It may also be sufficient to formulate only the shear moduli or viscosities as a function of the degree of cure.

Finally, the chosen atmosphere during photopolymerisation influences significantly the crosslinking speed. Previous work shows that without purge gas the crosslinking reaction is much slower [34]. Since available 3D printers usually work without a protective atmosphere of nitrogen, photo-DSC measurements must be carried out without purge gas. 


\section{Acknowledgement}

The financial support of the project "Constitutive modeling of UV-curing printed polymer composites" by the German Research Foundation (DFG) and Agence nationale de la recherche (ANR) under the grant numbers LI 696/20-1 and ANR-18-CE92-0002-01 is gratefully acknowledged.

\section{References}

[1] T. Kayhart, S. Shpiner, Analysis of Engineered Polymer Structures for Blunt Impact Protection, Technical Report, Predictive Engineering, Inc., 2018.

[2] J. Mueller, K. H. Matlack, K. Shea, C. Daraio, Energy absorption properties of periodic and stochastic 3d lattice materials, Advanced Theory and Simulations (2019) 1900081.

[3] D. Gailevičius, V. Padolskytè, L. Mikoliūnaitè, S. Šakirzanovas, S. Juodkazis, M. Malinauskas, Additivemanufacturing of $3 \mathrm{~d}$ glass-ceramics down to nanoscale resolution, Nanoscale Horiz. 4 (2019) 647-651.

[4] A. Bhargav, V. Sanjairaj, V. Rosa, L. W. Feng, J. F. Yh, Applications of additive manufacturing in detistry: A review, Journal of Biomedical Materials Research 106 (2018) 2058-2064.

[5] Spotlight on Additive Manufacturing, Technical Report, Ansys, Inc., 2018.

[6] Additive Manufacturing for Generative Design, Technical Report, Dassault Systèmes, 2017.

[7] Determining cure profile and post-cure shrinkage of photopolymers using UV accessory on a rotational rheometer, Technical Report, Malvern Instruments Limited, 2015.

[8] E. Skliutas, S. Kasetaite, L. Jonušauskas, J. Ostrauskaite, M. Malinauskas, Photosensitive naturally derived resins toward optical 3-d printing, Optical Engineering 57 (2018) $1-9$.

[9] C. Liebl, M. Johlitz, B. Yagimli, A. Lion, Three-dimensional chemo-thermomechanically coupled simulation of curing adhesives including viscoplasticity and chemical shrinkage, Computational Mechanics 49 (2012) 603-615.

[10] M. Hossain, G. Possart, P. Steinmann, A small-strain model to simulate the curing of thermosets, Computational Mechanics 43 (2009) 769-779.

[11] M. R. Kamal, S. Sourour, Kinetics and Thermal Characterization of Thermoset Cure, Polymer Engineering and Science 13 (1973) 59-64.

[12] M. R. Kamal, Thermoset Characterization for Moldability Analysis, Polymer Engineering and Science 14 (1974) 231239.

[13] S. Kolmeder, A. Lion, R. Landgraf, J. Ihlemann, Thermophysical properties and material modelling of acrylic bone cements used in vertebroplasty, Journal of Thermal Analysis and Calorimetry 105 (2011) 705-718.

[14] R. Mahnken, Thermodynamic consistent modeling of polymer curing coupled to visco-elasticity at large strains, International Journal of Solids and Structures 50 (2013) 20032021.

[15] R. Mahnken, C. Dammann, A three-scale framework for fibre-reinforce-polymer curing part i: Microscopic modeling and mesoscopic effective properties, International Journal of Solids and Structures 100-101 (2016) 341-355.

[16] M. Hossain, P. Steinmann, Continuum physics of materials with time-dependent properties: Reviewing the case of polymer curing, Advances in Applied Mechanics 48 (2015) 141-259.

[17] G. R. Tryson, A. R. Shultz, A calorimetric study of acrylate photopolymerization, Journal of Polymer Science Part B: Polymer Physics 17 (1979) 2059-2075.

[18] A. Maffezzoli, R. Terzi, Effect of irraditation intensity on the isothermal photopolymerization kinetics of acrylic resins for stereolithography, Thermochimica Acta 321 (1998) 111121.

[19] P. J. da Silva Bartolo, Photo-curing modelling: direct irradiation, The International Journal of Advanced Manufacturing Technology 32 (2007) 480-491.

[20] J. Wu, Z. Zhao, C. M. Hamel, X. Mu, X. Kuang, Z. Guo, H. J. Qi, Evolution of material properties during free radical photopolymerization, Journal of the Mechanics and Physics of Solids 112 (2018) 25-49.

[21] C. Mendes-Felipe, D. Patrocinio, J. M. Laza, L. Ruiz-Rubio, J. L. Vilas, Evaluation of postcuring process on the thermal and mechanical properties of the Clear02 $2^{\mathrm{TM}}$ resin used in stereolithography, Polymer Testing 72 (2018) 115-121.

[22] G. W. H. Höhne, W. F. Hemminger, H.-J. Flammersheim, Differential Scanning Calorimetry, 2003. doi:10. 1007/978-3-662-06710-9.

[23] R. M. Christensen, Theory of Viscoelasticity. An Introduction, 1982. doi:10.1016/B978-0-12-174252-2.X5001-7.

[24] M. Hossain, P. Steinmann, Degree of cure-dependent modelling for polymer curing processes at small-strain. part i: consistent reformulation, Computational Mechanics 53 (2014) 777-787.

[25] A. Lion, P. Höfer, On the phenomenological representation of curing phenomena in continuum mechanics, Arch. Mech. 59 (2007) 59-89.

[26] T. Sain, K. Loeffel, S. Chester, A thermo-chemomechanically coupled constitutive model for curing of glassy polymers, Journal of the Mechanics and Physics of Solids 116 (2018) 267-289.

[27] B. Yagimli, A. Lion, Experimental investigations and material modelling of curing processes under small deformations, Journal of Applied Mathematics and Mechanics 91 (2011) 342-359.

[28] N. Stander, W. Roux, A. Basudhar, T. Eggleston, T. Goel, K. Craig, LS-OPT ${ }^{\circledR}$ User's Manual, Livermore Software Technology Corporation, 2015.

[29] J. Fournier, G. Williams, C. Duch, G. A. Aldrige, Changes in Molecular Dynamics during Bulk Polymerization of an Epoxide-Amine System As Studied by Dielectric Relaxation Spectroscopy, Macromolecules 29 (1996) 7097-7107.

[30] C. Jochum, M. Arrigoni, M. Boustie, J.-C. Grandidier, A cut-off fracture approach for residual stress estimation in thick epoxies, Materials Science \& Engineering Technology 47 (2016) 530-538.

[31] R. Landgraf, J. Ihlemann, Thermally Controlled Adhesive Curing during the Production of Piezo-Metal-Compounds: Finite Element Modeling and Analyses, Advanced Engineering Materials 20 (2018) 1800411:1-1800411:9.

[32] C. Leistner, S. Hartmann, D. Abliz, G. Ziegmann, Modeling and simulation of the curing process of epoxy resins using finite elements, Continuum Mechanics and Thermodynamics 29 (2018) 1-24.

[33] A. T. DiBenedetto, Prediction of the Glass Transition Temperature of Polymers: A Model Based on the Principle of Corresponding States, Journal of Polymer Science Part B: Polymer Physics 25 (1987) 1949-1969.

[34] M. C. Rusu, C. Block, G. Van Assche, B. Van Mele, Influence of temperature and UV intensity on photopolymerization reaction studied by photo-DSC, Journal of Thermal Analysis and Calorimetry 110 (2012) 287-294. 